\title{
New Properties of Crimes in Virtual Environments
}

\author{
Roman Dremliuga ${ }^{1, *}$, Natalia Prisekina ${ }^{1}$, Andrei Yakovenko ${ }^{2}$ \\ ${ }^{1}$ School of Law, Far Eastern Federal University, Vladivostok, 690922, Russia
}

${ }^{2}$ National Technology Initiative Center for Neurotechnology, Virtual and Augmented Reality Technologies, Far Eastern Federal University, Vladivostok, 690922, Russia

\begin{tabular}{l} 
A R T I C L E I N F O \\
\hline Article history: \\
Received: 27 August, 2020 \\
Accepted: 04 December, 2020 \\
Online: 28 December, 2020 \\
\hline Keywords: \\
Virtual reality \\
Criminal law \\
Virtual crime \\
\end{tabular}

\begin{abstract}
A B S T R A C T
Virtual reality is a technology that literally allows constructing of a new reality for its users. $V R$ has huge potential, it is able to change social life, communications, and the academic sphere, but VR may also be applied by criminals. In the present paper properties of potentially committed crimes in VR are analyzed. It is concluded that VR provides criminals with some advantages. The first advantage is the multijurisdictional problem in prosecuting the offender that is usual for most of IT. The second advantage is that a virtual environment gives a realistic experience to the user and literally replaces the real world. The third advantage is an integration of virtual-world environments with haptic devices that may factually affect the user in the real world. All of these allow the commission of new kinds of crimes where properties of conventional and cybercrimes are combined. Among such kinds of crimes analyzed in the paper are cybercrimes that affect the body and mental state of victims. $V R$ is researched as a first tool that in combination with haptic devices gives criminals the opportunity to commit crimes against sexual freedom and to cause physical harm. Thus, VR crimes need a special legal framework that will take into consideration this kind of crime.
\end{abstract}

\section{Introduction}

This paper is an extension of work originally presented in 2019 International Conference on Cybersecurity (ICoCSec) [1]. Virtual reality (VR) is one of the most promising breakthroughs. It literally replaces the real world. VR dips humans into an alternative reality where different aspects of human life are imitated. The tasks of introduction of any information technology may vary, for instance, technology could be applied to make the world around us better. At the time of the pandemic, it is the only way to visit new spaces that are so similar to the real world [2]. Virtual reality is not a single technology but a set of technologies that permit a developer to construct an environment that would be recognized by our body and mind as real. VR is a tool to create new realistic worlds. Sometimes this environment is used for work, very often for gaming and social interaction. There are some forecasts that soon people will prefer virtual worlds over reality.

Virtual reality is a widely used technology. The market of VR is anticipated to grow from US\$27 billion in 2018 to US\$209.2

"Corresponding Author: Roman Dremliuga, Email: dremliuga.ri@dvfu.ru billion in 2022 [3]. Some researchers noted that VR attracts a lot of investors' attention and large companies invest in the technology [4].

Virtual reality is technology that literally allows constructing a new reality for its users. VR has great potential, it is able to change our everyday life, communications, and academic sphere. Nowadays virtual reality is actively applied in different spheres. The biggest driver is the entrainment and computer games. Also, a VR application is noticeably beneficial in teaching $[5,6]$. Sometimes virtual reality helps to simulate real situations [7]. VR is widely applied in medicine [8]. Some cases of rehabilitation of felons in VR exist $[9,10]$. In addition, a virtual world can be tuned in accordance with the needs of the user that makes it more attractive than real.

Virtual reality technology is a variety of hardware and software tools, which help to imitate a real or fictional world for a user. It interacts with our senses (vision, hearing, touch, smell, sense of balance, etc.) and misleads the body and mind that they are in the real world [11]. Virtual reality is an environment that is imitated through technology. The main virtual reality device is a special 
helmet, that may mimic the outside world through rendering video and audio. There are also numerous additional devices such as controllers, joysticks, steering wheels, haptic interfaces which increase a realism of VR illusion. All devices are designed to make an imitated world as real as possible.

"Virtual reality" has a common-used definition of the term. It is considered that VR is an information technology with special characteristics. For example, the following definition is suitable for the goals of the research. Virtual reality term is interpreted as "a technically constructed interactive environment with the help of computer facilities; as the generation and operation of objects, real or imaginary, on the basis of their graphic representation, simulation of their physical properties and ability to influence and independent presence in space, as well as the creation of such objects by means of special computer equipment" [12].

Development and introduction of any technology depend on a legal framework that is applied. Investors, developers, and users have to understand current and future legal rules that concern application of technology. In terms of business, the legal expertise is necessary on the step of making the initial decision. Wrong assessment of legal risks could lead to a total misapplication of funds, time, and efforts. Thus, to understand development of VR it is necessary to understand the current and future legal frameworks in this sphere.

The question is whether VR is another technology to which existing cyberspace laws apply or whether entirely new rules need to be developed. In order to form the correct legal framework, it is necessary to understand the conceptual differences between this technology and other technologies.

The sufficient legal risk is to face criminal cases with new properties. Some technologies provide tremendous opportunities for commitment of crimes. If a developer understands which challenges would exist in terms of criminal application, he will design his products by taking into consideration potential risks. The key is understanding how VR could be used by criminals and how it could be treated from a legal point of view.

The idea of this paper is not directly to enumerate possibilities of illegal use of VR but assess future prevalence and social consequences. To achieve this goal, it is necessary to utilize some assumptions that research will rely on.

- If VR maintains to achieve the same illegal result as some other technology, this automation will be replaced by VR in case of higher effectiveness of VR.

- If some property bolsters to spread VR use in society, this property will be the key characteristic that makes VR useful for criminals.

- Enforcement is always behind leaders of the crime 'industry' since it is bound by strict regulation and bureaucracy.

Thus, three aspects were carried out in the present paper. Properties of VR as a tool of crime were analyzed; challenges for the development of regulation that is caused by the characteristics of VR were reviewed. Some recommendations for the development of a legal framework were provided.

\section{Research methodology}

Research methodology for studying legal challenges of VR technology that goes beyond both dogmatic analysis has to be applied in the research. Analysis in the research follows positions developed within the philosophy of law and legal theory. Among relevant methodologies are the hermeneutic and argumentative approaches.

As the research is interdisciplinary, there is a synergy between methodlogies of disciplines. Interdisciplinary research must extend beyond mere multiplication of disciplinary methods and perspectives, to incorporate some element of integration or, mutual dialogue between disciplines. Interdisciplinarity is absolutely essential for the research as its object lies on the intersection of disciplines. As fairly commented some authors "it is important to have a multidisciplinary approach inconstructing such a methodology as the tools analysed stem from different disciplines and domain of expertise" [13].

Factually the research is based on a comparison of current and future properties of VR technology with current and potential legal framework. The focus on the legal and societal impacts of VR use requires us to observe the results of researches in the sphere of influence of virtual environment on humans. It is clear that such an important question cannot be accepted only on the basis of one or several studies, therefore, it is necessary to use the results of a whole set of studies to draw conclusions about the characteristics of VR, important for the formation of legal norms for its regulation.

The expected results of the study are directions where the legal framework applied to VR has to be upgraded. Recommendations with a high level of abstract should be developed. In future, de lege ferenda proposals could be based on our results.

Despite analysis and conclusions of the paper could be applied in different legal systems, the study is mostly focused on legal practice and conceptions of the United States and EU states as leaders in introduction of VR. The reseacrh is less applicable to asian technological leaders due to sufficicent differences in legal frameworks. Some ways of VR applications that are usual in the United States and EU are prohibited in other countries. For instance, virtual sex or VR pornograhpy are illegal in China, Indonesia and Malaysia.

Thus, the methodology can be shortly described as the application of a legal case study to the research of technology with an enormous social impact. The paper as some others [14] utilized a case study methodology. It is manifested in gathering and analyzing proven effects of VR on a user in terms of social, ethical, and legal risks. As the area of risk is quite wide, the paper focuses on risks related to the commission of criminal offences in VR.

\section{Legal challenges}

\subsection{Increasing significance of $V R$}

The main purpose of VR is to provide an environment for spending time. The application of VR for gaming dominates nowadays but VR is also used for workshops and business meetings. It is actively applied for educational purposes and simulation of difficult tasks. The advantage of VR is clear: a 
customized reality that provides a high level of similarity of human reactions with the real nature. It is effective for games where people pass through all spectrum of emotions as well as for training where people become ready for the stress of a real situation. Most VR applications in business communications or games imply audio and video information exchange in some virtual space that increases the level of participation.

The level of realism could be even improved when real appearance is applied. In virtual reality, a user is frequently seen by others as an anthropomorphic avatar whose appearance may be similar to the user's appearance in the real world or may be completely different. Despite the voice and personal appearance of the virtual world can be altered, this does not exclude the possibility that voice and visual appearance of the user would be the same as in the real world.

The big advantage of VR is that people are able to communicate in a realistic way even if they are separated in the real space. Thus, people, in the form of relatively realistic avatars, communicate as they would be face-to-face. VR provides the possibility to imitate eye contact and to manipulate virtual objects that participants see together. It is similar to a video call, but participants of VR meetings have a higher level of involvement.

VR technology is far from perfection. Headsets cannot track eye movement with high accuracy. VR Graphics is far from a full imitation of the representation of the real world and is rather applied to construct some simple spaces with a low quantity of details. Facial expressions are still rendered in virtual reality quite poorly.

Despite the above, virtual reality is a very popular environment for spending time and human interaction. Under different studies, the amount of VR's application for spending time and social interaction is fastly growing [15]. There are approximately 170 million users of virtual reality worldwide. A double increase in the number of VR consumers is evidenced in recent years.

The growth of legal applications of VR implies an increase in criminal use. It is anticipated that the number of virtual reality users should correlate with the number of crimes committed in VR, as this was observed with the growth of the number of Internet users. As more people use VR worldwide, there are more potential victims and criminals in VR. Some researchers found the proliferation of the use of the global network (the Internet) as a major factor in the growth of cybercrime. Thus, the number of criminal acts in VR is expected to increase due to the growth of the number of its users [16].

Wide use of VR leads to a classic dilemma. A legislator has to choose between the need for a free exchange of computer information, as a factor of digital economy growth and the need to defend state and social security by restrictions on IT use. To find the balance between public security and the necessary freedom of technology application for its development, a lot of different factors have to be taken into consideration.

As with any technology, VR may facilitate criminal activity. Virtual environment may be a tool or instrument to commit a crime as well as an additional factor that facilitates it. Recently, the use of high technology to commit crimes increases, and it concerns virtual reality. An opportunity how criminal law is applicable in case of crime in virtual environment should be studied.

\subsection{VR as a potential tool to commit a crime}

VR is in some sense a unique technology that makes crimes with the application of that technology dissimilar with others. It may constitute difficulties for the investigations of such offences. VR as a tool confers crimes by new properties. Virtual reality is not simply another new technology, which requires to design technical standards. Rather VR application requires to introduce a new legal framework. There are several reasons why a traditional legal framework does not qualify. Basically, due to its special characteristics, VR requires fundamentally new legal frameworks and solutions.

The first, commonly known problem is cross border jurisdiction. It is difficult to identify a person with whom the user interacts in VR. Virtual environment and its users are often in different jurisdictions. A world that is undivided in VR may exist regardless of borders of the concrete state in terms of its users. Millions VR users have no idea where the virtual reality platform is physically located, where their data are processed and stored. Thus, virtual space may be outside the jurisdiction of the state where the victim applies to law enforcement.

Some authors commented that: "It will take a lot to get domestic police interested in investigating a crime where the criminal is in a foreign country. ... Still, the greater difficulties of extradition are likely to exceed the greater ease of proof. And many VR street crimes might thus be practically ignored by traditional police departments" [17]. Thus, the multijurisdictional challenge is the multidimensional problem. There are a lot of questions as from legal assessment of committed offence as well from criminal procedure rules.

Any digital cross border space poses a challenge to law enforcement and the legislator. People from states with different legal systems meet in a virtual environment. Cross border character of VR spaces creates a situation where the crime has anonymous, remote in space, and global character. When virtual reality is applied to commit a crime, deanonymization and pursuing criminals become very difficult and even impossible.

The second feature of virtual reality as a place, tool, or instrument of crime is the capability of a virtual environment to create an illusion of reality. Sometimes this property forces real consequences for the potential victims of crime in VR.

Studies demonstrate that people uploaded at VR may be afraid of walking across a wooden plank that is on the ground in the real world but placed in virtual reality between roofs of skyscrapers. The experimental environment forces similar psycho-emotional reactions of a probationer as if it happens in the real world [18]. Dizziness, nausea, stomachache, and shaking body are just a few of the reactions to virtual heights [17]. Thus, experience in a virtual environment, in turn, may bring real consequences for body and mind. The VR customer may be literally frightened to a state of heart attack by a game in which he (she) feels quite real [19]. In case of the absence of consequences for the body, it may be a difficult psychologically traumatic experience. Thus, a virtual experience could cause permanent physical or psychological harm.

The realism of virtual environments has also positive effects, for example, VR is successfully applied for autism control [20] or in treatment of phobias [21]. But the side effect of VR realism is 
that it is a perfect tool for hackers or other cybercriminals to "hack" into the victim's heads. Earlier, cybercriminals only had distant access to property, business reputation, and other values, but now they can literally get inside the brain. Harm also could be caused by the application of VR in medical treatment without direct intention [22].

In comparison with the real world, VR provides certain advantages for criminals in terms of the range of psycho-emotional reactions that could be provoked. In the physical world, it is very difficult to place a victim into the "Grand Canyon" and is even impossible to organize a meeting with a monster from science fiction. Hence, it is possible to distantly manipulate consciousness and emotions through VR by imitating any real or fictional environment. [23]. The character of such intervention is limited only by the imagination of the criminals. Current studies demonstrate that with the help of VR any emotion for up to 8 minutes could be forced [24].

Virtual worlds become more realistic due to the development of VR software and hardware. Objects are rendered with a higher degree of detail. More sophisticated models are applied to imitate interaction between objects in virtual reality. The link between events in VR and the body of the user gets more channels. Some researchers underline that higher realism implies a more significant psycho-emotional response of the customer on events in the computer environment [25].

As some researchers claim "Multimodal inputs will be combined in unprecedented patterns of stimulation that will ultimately make a VR experience more real than reality. Strong social emotions, in turn, will likely reinforce the sense of presence and make it easier to ascribe mental states to other virtual characters" [26]. Thus, intervention into human mental state is conceivably even more aggressive in future because new developments in sphere of VR technology.

The next challenge is development of haptic devices or other devices that are capable to affect the customer in the reality. Haptic technology enhances, new ones appear each year [27]. For example, there are enough investments in technologies for remote sexual intercourse. In the case of virtual environment application, skin sensations are networked between the users. The widespread use of such technologies implies that a criminal is potentially able to commit illegal intimate contact.

In some studies, conducted in the United States, it is underlined that hacker's replacement of another user in a sexual act or unauthorized access to the user's body in VR with the use of a haptic interface has similar characteristics with crimes against sexual freedom. In the legal research papers, such act committed with intention is considered to be either sexual harassment or rape [28]. Before haptic technology, it would be impossible to commit a rape distantly. Thus, haptic devices and similar equipment provide the capability to intervene in body inviolability.

The opinion exists that the above challenge could be overcome by a technical solution. Some authors state that "the ability to define default consent in software can make VR safer than the real world-for instance, well-designed software may let me consent in advance to certain types of touching but not others, or touching by some people but not others, and touching that isn't consented to won't even be felt. The question that remains is to what extent, if at all, it should be viewed as the victim's job to set software consent boundaries; more on that below" [17]. Thus, the questions here: who would be responsible for the introduction of defense from body encroachments, what would be the liability of the developer or the seller for negligent informing about such kind of risk? And the main question is whether it is still crime or not?

All discussed above presuppose that VR allows to committing crimes which are impossible to commit with traditional IT. For instance, homicide could be committed on the Internet without VR, but not rape. A victim who has been raped in VR may not immediately realize what happens. Thus, a victim may be in a helpless state. As the victim is unable to understand that some illegal actions happen, he (she) is unable to take measures against the criminal. In some sense, it implies that rape or harassment in $\mathrm{VR}$ is probably even more dangerous because it is latent even for the victim.

Moreover, software and hardware for distant sex have low security against cyberattacks [29]. Often developers in this sphere are focused on new user engagement than on fixing vulnerabilities. It indicates that such kind of products could be easily hacked. Potentially criminals even could provoke VR users to move outside their secure boundaries in the reality through application of malware [30].

Due to the innovations in the VR sphere criminals are able to encroach not only on the mind but also on the body of victims. It is a complicated question whether the sexual assault in VR is comparable with real-world crime in terms of consequences for a victim. Of note, in the case of VR, there is a great number of difficulties in the prosecution of the offender. In addition, criminal significantly reduces the sum of risks related to the act (risk to be caught at the crime scene, risk of suffering if the victim resists, risk of public censure even if the guilt is not proven, and some others).

Development of haptic suits with feedback poses a risk of increasing harm-related crimes. The suit by electrical impulses informs the body of what happens with it in VR. The haptic device stimulates skin when some object touches the user in virtual world. Currently, there are no regulations on the permissible power of impact on the body. It is not legally defined what the degree of pain and harm is possible in VR. Of note, such a rule has to depend on the individual characteristics and other parameters. Due to the absence of regulation a risk of inflicting real suffering from haptic suits in virtual reality exists.

For instance, «new peripherals and technologies enter the market supporting a variety of interaction fidelities, such as data glove controllers, skeletal motion capture suits, tracking systems, and haptic devices» [31].

\subsection{Immoral acts}

As described above some manifestations of VR are not covered by modern legal regulations but there are some difficulties in addressing the mentioned challenges even in terms of moral rules. As law and morality are both means of conducting control, they are strongly linked. It does not imply that both social regulators have to correlate each other, but at least legislator has to take into consideration the norms of molality. And if a gap in the legal framework exists, VR has to be assessed from a moral point of view. A moral system of norms is clearly often very dynamic and opaque, but it is often helpful to know what is good or what is bad from a moral point of view. Moreover, the last social initiative in countries of Western Europe and Northern America as MeToo and 
BLM demonstrate that moral norms could have even bigger impact on society than legal rules.

The first question is arising from identity flexibility in a virtual environment. In VR any user can choose any appearance that is provided by a software, risk exists that a person will apply the wrong appearance. At the same time, avatars and names influence on the perception of the user by other persons. These characteristics that are very significant for the attitude of other persons could be easily changed. It raises some ethical questions that are significant from ethical and sometimes legal point of view.

Imagine a teenager who uses VR poses as an adult and engages in an intimate conversation or even virtual sexual contact with an adult person. Who would be liable for such actions? Who has to take precautionary measures? Who must be in charge of the customer's appearance? What would the legal consequences of similar action be? Probably the legislator has to oblige VR developers to incorporate technical solutions for this kind of case. If one person misleads another person by his/her appearance in VR, how does it correspond with moral or even legal rules?

Even if users represent themselves in VR without misleading other persons, appearance also could be an issue. A demonstrative example is an avatar that looks like a naked body. Public nudity is banned by ethical and legal norms in most of the states, but a video demonstration of a naked body is rather not. Whether the naked body in VR is regarded as pubic nudity or is it a demonstration of video content from an ethical and legal perspective? Does the legislator have to oblige the developer to add measures against the use of such avatars?

A prosecution of public nudity in VR is harder from a legal and ethical point of view. What is a nature of this act? Factually it is a rendering of video content. However, a demonstration is accompanied by emulating personal presence. Thus, nudity in VR is not the same as nudity in games or movies. At the same time in terms of the intent of a person, it could be considered as public nudity. Virtual avatar is able to be real human size, with real human movements, seemingly standing close to other users. Mentioned avatars are perceived much more like a real person than a picture on a screen. Such actions may offend someone's feelings or cause psychological trauma in a demonstration for minors.

The issue of a proper avatar in VR is not limited to the nudity problem. For instance, an avatar may offend someone's feelings in other ways. It may be intolerant of gender, race, religion, political beliefs. More ways to offend or to cause suffering exist in VR. Imagine that someone's deceased relative's face is used in VR. Some users are able to use Hitler or famous serial killer's face for their avatars.

It is able to harm, disgust, or provoke users which see the avatar in VR. Appearance could be chosen as intentionally as well as recklessly. Developers can clearly provide technical measures to filter or block graphical representation of a user in virtual reality. But as it is linked with additional costs of software development, special regulation obliging VR companies has to be enacted.

Another problem is compliance with ethical norms applied in reality. As users all come to VR from the real world, some expectation exists on the conduct of the user in a virtual environment. There is an enormous temptation to transpose the moral norms of the ordinary world into the virtual world. Is it appropriate from a moral point of view to scream, create a mess, etc. in the VR art gallery?
There is a suggestion that "VR environment operators can easily implement code that can deal with the screamers. The operator could, for instance, allow each user to control the perceived volume, for that user, of any other user. That is good not just to silence the screamers, but also to quiet down acquaintances who are a bit too loud, or to amplify acquaintances who mutter. And this should be technically trivial to code" [17]. But it provokes the same question who will oblige the operator or the developer of the application to bear additional expenses on the implementation of these rules. Any state that implements such rules will automatically decrease chances in the race for technological dominance in the sphere of VR.

Of note, the issue of content filtering in VR is within the scope of the free speech agenda. When the legislator imposes additional legal requirements on VR developers, it implies reducing-or elimination-of the ability of individuals to speak freely in VR [32]. It is difficult to differentiate harmful behavior from an expression of someone's freedom of speech right on the level of development, as any case depends on context, viewer, or environment. Thus, a restriction for the developer or the operator is a threat to the freedom of speech in a virtual environment.

Another issue related to legal restrictions in VR is enforcement. The developer that designs or supports VR software or hardware is able to incorporate restrictions in its products; however, it is inevitably linked with real-time tracking of the user's conduct. It is presumably a high price for secure VR.

Development of restrictive measures will face also technical issues. Experience of companies which control and process of big data shows that "training moderators to overcome cultural biases or emotional reactions in the application of rules to facts can be analogized to training lawyers or judges. Regardless of the label, training content moderators involves a repetitive process to override cultural or emotional reactions and replace them with rational valid resolutions" [33]. So, an automatic realization of rules could produce unjust decisions, discriminative ruling, and other negative effects. It is probably more difficult to automatically make just decisions about the character of VR scenes than to create a transparent judicial system. This part of companies' work is not limited by the assertion that technical decisions have to be developed, the company has to create a division to manage disputes with customers which are not enjoyed by rule application.

Big IT companies are often criticized for a lot of power, but the capabilities of a virtual environment will allow private companies to have almost unlimited power over customers and their data. Factually, companies get control over all channels of human perception in different spheres from gaming to sexual relations. "VR settings mimicking as close as possible the multisensory nature of real-world human interactions would be a welcome and powerful addition to the field. Experiments aligning auditory, vestibular, and tactile signals with the visual domain are already improving VR immersion and realism" [26]. Thus, some private companies will control our reality, minds, and bodies.

\section{Discussion}

The study is just the beginning. This paper describes the key challenges related to managing risks of virtual environment use. VR is in the spotlight of social attention and attracts more attention from researchers. The study could be developed in different directions. There are several topics for further research. First of all, 
it is necessary to systematize theoretical and practical legal knowledge related to the regulation of VR. It could include but not be limited to the research literature, legal practice, and international public agreements, as well as case-study.

Also, it is necessary to define the term "VR" and differentiate its categories and types, depending on the depth of immersion. It is helpful in terms of systematization and unification of results of current and future studies. It is necessary to categorize types of crimes that could be committed in VR. As some authors fairly comment on significance of legal definition and categorization of VR "it will serve as a basis for choosing the optimum regime of their legal protection" [34].

In terms of multidisciplinary dialogue, it is necessary to find out categories of the permissible technical parameters of VR functioning. It is very supportive in terms of preventing the destructive impact of technology on the psychophysiological state of the subject. For instance, there is a need to regulate the frame rate to avoid: headache, nausea, dizziness, epilepsy, and other disorders. It is especially critical for the application of VR by minors and juveniles.

Some authors claim that it is necessary "to add additional investigation and analysis testing stages to the development of virtual reality technologies in efforts to protect the public. These tests might not focus on physical health and safety concerns, but rather on physiological and social influences" [35]. However, in accordance with the analysis, the main concerns are mental and physical health. Moreover, any measures that take into consideration social influences would be very local and depend on the volatile social environment.

There is a need to define and regulate the allowable limits of the subject's functional permissibility in virtual reality (virtual ethical standard). Moreover, it is necessary to study the potential consequences of non-compliance with the prescribed rules of conduct, in order to prevent the direct projection of actions in VR in real life, such as the loss of boundaries of objective reality and the unconscious killing of a person in the real world.

In some papers, authors conclude that current law is very flexible and could be also applied to VR [22]. These studies do not take into consideration the dual nature of VR, i.e. this cybertechnology is rapidly merged with real world. The question arises in this study that current law is not applicable in cases where VR manifests itself in cyberspace and space of the real world. For instance, illegal access to haptic devices is not the same as usual "hacking".

There is research on restrictions of speech in VR in terms of US legislation. However, there is no conclusion how it corresponds to the First amendment and leaves a lot of space for further discussion [36]. In accordance with the analysis of current research in the sphere of medicine and psychology, it is difficult to conclude whether it is appropriate to apply the law of real world or virtual worlds for VR. The main reason is that virtual space and behavior of users there are often perceived as reality by other users.

In addition, there are several problems related to VR regulation that have to fall within the scope of criminal law. For instance, rules for the regulation of rendering in VR, application of real-world standards in VR, total control of users' conduct by IT giants, and appearance that misleads other users.

\section{Conclusion}

In summary, our study demonstrates that the offender gets new opportunities in VR. It literally helps criminals to hack the head or body of the victim. VR allows to provoke the emotions and to manipulate the consciousness of the victim at a completely new level. It is proved in the research that VR use could not be regulated as any other IT technology as the effect of perception by the victim is comparable in strength with the effect of events in the real world. In the paper characteristics of the virtual environment that have to be taken into consideration during a process of VR, regulation development is underlined.

Moreover, VR combined with haptic devices requires even more sophisticated regulation as such environment merges with the real world. Due to the integration of skin-feedback devices into the virtual environment, the consequences of VR's actions also have an influence on the user in the real world that could not be considered as just illegal manipulation with computer information. As many types of criminal acts with harm to the victim's body can now be committed remotely, a new kind of crime with dual nature is originated.

The article shows that there are not enough criminal law measures to combat crimes in VR. First of all, the legislation should provide for the obligations of the developer of VR technologies in order to minimize the possibility of criminals. Development and adoption of such measures is not an easy task, it is necessary to minimize economic costs in the implementation of these measures, and to prevent serious restriction of freedom of speech.

\section{Conflict of Interest}

The authors declare no conflict of interest.

\section{Acknowledgement}

The study was carried out with the financial support of the Russian Foundation for Basic Research in the framework of research project No. 18-29-16129

\section{References}

[1] R. Dremliuga, R. Iakovenko, N. Prisekina "Crime in virtual reality: Discussion", 2019 International Conference on Cybersecurity, ICoCSec 2019, 8970947, 81-85, 2019.

[2] A.O.J. Kwok, S.G.M. Koh "COVID-19 and Extended Reality (XR)", $\begin{array}{llll}\text { Current Issues } & \text { in } & \text { Tourism, }\end{array}$ https://www.tandfonline.com/doi/full/10.1080/13683500.2020.1798896

[3] Forecast augmented (AR) and virtual reality (VR) market size worldwide from 2016 to 2022 (in billion U.S. dollars). Statista, 2018. Retrieved October 26, 2020 from https:/www.statista.com/statistics/591181/globalaugmented-virtual-reality-market-size/.

[4] M. Meißner, J. Pfeiffer, C. Peukert, H. Dietrich, T. Pfeiffer "How virtual reality affects consumer choice", Journal of Business Research, 117, 219. 231, 2020.

[5] M. Figueiredo, R. Mafalda, A. Kamensky "Virtual reality as an educational tool for elementary school", Smart Innovation, Systems and Technologies, 198 SIST, 261-267, 2021.

[6] Y.P. Zinchenko, P.P. Khoroshikh, A.A. Sergievich, S.A. Gutnikov, K.S. Golokhvast "Virtual reality is more efficient in learning human heart anatomy especially for subjects with low baseline knowledge", New Ideas in 
Psychology, 59,100786, 2020. DOI: 10.1016/j.newideapsych.2020.100786

[7] S. Ahn, J. Bostick, E. Ogle, K.L. Nowak, K. McGillicuddy, J. Bailenson "Experiencing Nature: Embodying Animals in Immersive Virtual Environments Increases Inclusion of Nature in Self and Involvement with Nature", Journal of Computer-Mediated Communication, 21(6), 399-419, 2016. https://doi.org/10.1111/jcc4.12173

[8] M.C. Howard "A meta-analysis and systematic literature review of virtual reality rehabilitation programs", Computers in Human Behavior, 70(1), 317 327, 2017.

[9] B. Ticknor Virtual Reality and Correctional Rehabilitation: A Game Changer", Criminal Justice and Behavior, 46(9), 1319-1336, 2019.

[10] B. Ticknor "Using virtual reality to treat offenders: An examination", International Journal of Criminal Justice Sciences, 13(2), 316-325, 2018

[11] G.P. Otjutsky "Understanding the virtual reality: methodological difficulties", Historical, philosophical, political and legal sciences, cultural studies and art criticism. Issues of theory and practice, 2-2 (52), 161-170, 2015.

[12] E.A. Solodkina "To the definition of the concept of virtual reality", Bulletin of the Peoples' Friendship University of Russia. Series: Philosophy, (1), 190194, 2004

[13] V. Jaillot, M. Istasse, S. Servigne, M. Rautenberg, I. Lefort, "Describing, comparing and analysing digital urban heritage tools: A methodology designed with a multidisciplinary approach", Digital Applications in Archaeology and Cultural Heritage, 17,e00135, 2020.

[14] P.P. Shrestha, B. Davis, G.M. Gad, "Investigation of Legal Issues in Construction-Manager-at-Risk Projects: Case Study of Airport Projects", Journal of Legal Affairs and Dispute Resolution in Engineering and Construction, 2(3),04520022, 2020.

[15] E. Fritsch "Growth in wearable technologies", Screen Printing, 110(5), 2226, 2019.

[16] R. Dremliuga "Subculture of Hackers in Russia", Asian Social Science, 10(18), 158-162, 2014. DOI: 10.5539/ASS.V10N18P158

[17] Mark A .Lemley, E. Volokh "Law, Virtual Reality, and Augmented Reality", University of Pennsylvania Law Review, 166, 1051-1138, 2018. Available at SSRN: https://ssrn.com/abstract $=2933867$ or http://dx.doi.org/10.2139/ssrn.2933867

[18] Q . Wang, F. Hu, H.Wang "Are you afraid of heights and suitable for working at height?", Biomedical Signal Processing and Control, 52, 23-31, 2019.

[19] T.W. Cleworth, R. Chua, J.T. Inglis, M.G. Carpenter "Influence of virtual height exposure on postural reactions to support surface translations", Gait and Posture, 47, 96-102, 2016.

[20] S.N.V. Yuan, H.H.S. Ip "Using virtual reality to train emotional and social skills in children with autism spectrum disorder", London Journal of Primary Care, 10(4), 110-112, 2018.

[21] J.-M. Park, G.-Y. Noh "The effects of vr simulation game on driving fear and efficacy", JP Journal of Heat and Mass Transfer, 15(Special Issue 2), 161-169, 2018.

[22] Y.Y. Lim, P. Poronnik, T. Usherwood, B. Reeve "Medical negligence laws and virtual reality in healthcare", Australian journal of general practice, 49(8), 525-529, 2020.

[23] R. Ueoka, A. AlMutawa, "Emotion hacking VR: Amplifying scary VR experience by accelerating actual heart rate", Lecture Notes in Computer Science (including subseries Lecture Notes in Artificial Intelligence and Lecture Notes in Bioinformatics), 10904 LNCS, 436-445, 2018.

[24] B. Meuleman, D. Rudrauf "Induction and profiling of strong multicomponential emotions in virtual reality", IEEE Transactions on Affective Computing, 2018, Available at: https://ieeexplore.ieee.org/document/8432120/

[25] J. Hvass, O. Larsen, K. Vendelbo "Visual realism and presence in a virtual reality game", 3DTV-Conference 2017-June, 1-4, 2018.

[26] A. Monti, S.M. Aglioti "Flesh and bone digital sociality: On how humans may go virtual", British Journal of Psychology, 109(3), 418-420, 2018.

[27] A. Leleve, T. McDaniel, C. Rossa "Haptic Training Simulation", Frontiers in virtual reality, 1(3), July 2020. https://doi.org/10.3389/frvir.2020.00003

[28] L. Strikwerda "Present and Future Instances of Virtual Rape in Light of Three Categories of Legal Philosophical Theories on Rape", Philosophy and Technology, 28(4), 491-510, 2015.

[29] M.J. Faustino "Rebooting an Old Script by New Means: Teledildonics-The Technological Return to the "Coital Imperative'", Sexuality and Culture, 22(1), 243-257, 2018.

[30] M.U. Rafique, S.-C.S. Cheung "Tracking Attacks on Virtual Reality Systems", IEEE Consumer Electronics Magazine, 9(2), 8977814, 41-46, 2020
[31] D. Duggan, C. Kingsley, M. Mazzeo, M. Jenkins "Exploring Extended Reality as a Simulation Training Tool Through Naturalistic Interactions and Enhanced Immersion", Lecture Notes in Computer Science (including subseries Lecture Notes in Artificial Intelligence and Lecture Notes in Bioinformatics), 11575 LNCS, 272-282, 2019.

[32] Jack M. Balkin, "Information Fiduciaries and the First Amendment", UC Davis Law Review, 49(4), 183-1234, 2016. Available at SSRN: https://ssrn.com/abstract=2675270.

[33] K. Klonick. "The new governors: The people, rules, and processes governing online speech", Harvard Law Review, 131(6), 1599-1670, 2018.

[34] T.A. Kochubey, "Legal protection of virtual and augmented reality technologies", Advances in Intelligent Systems and Computing, 1100 AISC, 184-191, 2020.

[35] B. Kenwright "Virtual Reality: Ethical Challenges and Dangers [Opinion]" IEEE Technology and Society Magazine, 37(4),8558774, 20-25, 2018.

[36] M.J. Blitz "The First Amendment, video games, and virtual reality training" in "Research Handbook on the Law of Virtual and Augmented Reality", Edited by W. Barfield and M.J. Blitz, Edward Elgar Publishing, 712 p.2018. 